\title{
A educação continuada como subsídio para a enfermagem no contexto do parto natural: uma revisão integrativa
}

\author{
Education continued as a subsidy for nursing in the context of natural children: an \\ integrative review
}

La educación continuada como subsidio a la enfermería en el contexto de los niños naturales: una revisión integradora

Selma Antunes Garcia1*, Juliane Nunes Falcão, Maria Luiza Rêgo Bezerra.

\section{RESUMO}

Objetivo: Avaliar a partir de produções bibliográficas recentes a importância da educação continuada utilizada como subsídio para enfermagem no contexto do parto natural. Métodos: Trata-se de uma revisão integrativa da literatura. As pesquisas dos artigos foram feitas entre janeiro a maio de 2021, nas bases de dados: LILACS, PUMED, SciELO, BDENF e BVS. Os critérios de inclusão utilizados foram: artigos de pesquisa publicados nos últimos cinco anos, artigos originais gratuitos e artigos em periódicos disponíveis nas bases de dados selecionadas, nos idiomas de português, inglês e espanhol. Após a busca e aplicação dos critérios de inclusão, foram selecionados 23 artigos para formulação da discussão. Resultados: $O$ enfermeiro exerce um papel relevante frente às ações educativas, a capacitação deste profissional colabora para a melhoria na assistência assegurando seu desempenho no cenário de assistência e gerencia. As categorias: Parto natural e a assistência de enfermagem; Defasagem na assistência: falta de educação continuada foram o foco da análise. Considerações finais: A educação continuada em enfermagem faculta na melhora da atuação prática e também pode promover uma qualidade assistencial no parto natural.

Palavras-chave: Parto natural, Enfermagem obstétrica, Educação continuada em enfermagem.

\begin{abstract}
Objective: Evaluate from recent bibliographic productions the importance of continuing education used as a subsidy for nursing in the context of natural childbirth. Methods: This is an integrative literature review. The articles were searched between January and May 2021, in the following databases: LILACS, PUMED, SciELO, BDENF and VHL. The inclusion criteria used were: research articles published in the last five years, free original articles and articles in journals available in the selected databases, in Portuguese, English and Spanish. After searching and applying the inclusion criteria, 23 articles were selected to formulate the discussion. Results: The nurse plays an important role in educational activities; the training of this professional collaborates to improve care, ensuring their performance in the care and management scenario. The categories: Natural childbirth and nursing care; Delay in care: lack of continuing education was the focus of the analysis. Final considerations: Continuing education in nursing facilitates the improvement of practical performance and can also promote quality of care in natural childbirth.
\end{abstract}

Key words: Natural childbirth, Obstetric nursing, Continuing education in nursing.

\section{RESUMEN}

Objetivo: Evaluar, con base en producciones bibliográficas recientes, la importancia de la educación continua utilizada como subsidio a la enfermería en el contexto del parto natural. Métodos: Se trata de una revisión

${ }^{1}$ Universidade Paulista (UNIP), Brasília - DF. *E-mail: selmagarcia22@gmail.com 
integradora de la literatura. Los artículos fueron buscados entre enero y mayo de 2021, en las siguientes bases de datos: LILACS, PUMED, SciELO, BDENF y VHL. Los criterios de inclusión utilizados fueron: artículos de investigación publicados en los últimos cinco años, artículos originales gratuitos y artículos en revistas disponibles en las bases de datos seleccionadas, en portugués, inglés y español. Luego de buscar y aplicar los criterios de inclusión, se seleccionaron 23 artículos para formular la discusión. Resultados: El enfermero juega un papel relevante en las actividades educativas, la formación de este profesional contribuye a la mejora en el cuidado, asegurando su desempeño en el escenario asistencial y de gestión. Las categorías: Parto natural y cuidados de enfermería; Retraso en la atención: la falta de educación continua fue el foco del análisis Consideraciones finales: La educación continua en enfermería facilita la mejora del desempeño práctico y también puede promover la calidad de la atención en el parto natural.

Palabras clave: Parto natural, Enfermería obstétrica, Educación continuada en enfermería.

\section{INTRODUÇÃO}

A Educação Continuada (EC) é uma metodologia que resolve atividades que implica na pratica e capacitação dos profissionais de enfermagem em atualização dos processos assistenciais e gerenciais. Isto posto, a educação continuada é um meio significativo de assegurar um cuidado de qualidade assistencial, o que pode ser evidenciado pela própria realidade brasileira que possui este método aplicável por meio da Política Nacional de Educação Permanente em Saúde, estabelecida pelo Ministério da Saúde mediante a legislação criada em 2007 (SILVA CPG, et al., 2020).

A EC é importante em todos os contextos da assistência e gerencia de Enfermagem, com destaque para a realidade do parto natural caracterizado como um evento que ocorre sem intervenções, onde não há o uso de procedimentos invasivos e farmacológicos para alívio de dor, estimulando a autonomia da mulher, possibilitando a liberdade para tomada de decisões (ARAÚJO BRO, et al., 2019; OLIVEIRA PS, et al., 2019). É um acontecimento culturalmente marcante na vida da parturiente e de seus parentes, onde deve ser preservada a autonomia e a percepção do nascimento de uma nova família, sendo assim, a enfermagem tem o dever de implementar as boas práticas no processo de parto conforme a Lei no 11.108 , de 7 de abril de 2005, que estabelece essas práticas e o direito a presença de um acompanhante de escolha da parturiente durante o pré-parto, parto e puerpério (SILVA EA, et al., 2021).

Em relação à incidência de parto normal, o Brasil ainda apresenta baixos índices de adesão a está prática, sendo a média de $38.2 \%$ a $38.6 \%$ segundo dados de 2017 a 2018. Historicamente, vivemos o processo invertido a respeito da conscientização do parto natural, onde a cesariana é vista como benéfica para saúde da mulher, sendo que é cientificamente comprovado que o parto natural apresenta mais benefícios tanto para mãe quanto para o bebê. O Brasil apresenta a segunda maior taxa de cesariana do mundo (FREITAS PF e FERNANDES TMB, 2016).

Neste tipo de parto ressaltam-se ainda mais a liberdade da mulher como prioridade, utilizando de técnicas naturais para alívio de desconforto como: técnicas de massagem, relaxamento e respiração, variação de postura banho de emersão e música (POSSATI AB, et al., 2017; ANDRADE LO, et al., 2017). Estas técnicas favorecem a progressão do feto, respeitando o corpo feminino e o parto como um evento da natureza, um processo fisiológico em que o próprio corpo trabalha a favor do nascimento (VILELA AT, et al., 2019).

Segundo a declaração de Alma-Ata, a Organização Mundial da Saúde incumbiu o Estado de promover ações estratégicas sociais acessíveis a comunidade implementando um sistema articulado de educação em saúde (QUENTAL LLC, et al., 2017). A enfermagem obstétrica apresenta um papel indispensável no tocante da educação em saúde, pois, é o profissional que mais acompanha o trabalho de parto, promovendo assistência desde o período gravídico até o puerpério com toda segurança, avaliando riscos e levando informação com qualidade (FREIRE HSS, et al., 2017).

É de extrema importância o acolhimento da parturiente pela equipe de enfermagem que são capazes de oferecer um atendimento de qualidade no cuidado à mulher no trabalho de parto. Ressaltando que a equipe 
de enfermagem é amparada pela Lei ㄲo 7.498 de 25 de junho de 1986 para atuar na atenção a mulher, parto e parto (LIRA IMS, et al., 2020). Todavia, esse acolhimento só será eficaz quando todos que constituem a equipe de enfermagem forem qualificados e preparados para essa pratica (SILVA TPR, et al., 2019).

Contudo, está revisão integrativa de literatura teve o objetivo de conceituar a importância da educação continuada para o parto normal, já que a mesma permite uma capacitação teórica e prática, sendo assim destacando incentivos que facilitem o acesso a EC, dispondo assim uma rotina com qualidade na assistência perante o parto normal.

\section{MÉTODOS}

\section{Tipo de estudo}

Trata-se de uma revisão integrativa da literatura que consiste numa sistemática que promove a fusão de informações e a admissão da aplicabilidade de resultados relevantes e prática (SOUZA MT, et al., 2010).

\section{Coleta e organização de dados}

A coleta de dados foi realizada de janeiro a maio de 2021, nas bases de dados: Literatura Latino-Americana e do Caribe em Ciências de Saúde (LILACS), Publisher Medline (PUBMED), Scientific Eletronic Library Online (SciELO), Bases de Dados de Enfermagem (BDENF) e Biblioteca Virtual em Saúde (BVS), utilizando os seguintes descritores e palavras-chave: Parto Natural, Enfermagem Obstétrica e Educação Continuada em Enfermagem.

Foram elaboradas combinações entre os descritores e/ou palavras-chave que originaram as estratégias de busca descritas no Quadro 1.

Quadro 1 - Descritores usados na pesquisa das bases de dados, 2021.

\begin{tabular}{|l|l|}
\hline Base de Dados & \multicolumn{1}{|c|}{ Descritores } \\
\hline $\begin{array}{l}\text { LILACS } \\
\text { BDENF }\end{array}$ & $\begin{array}{l}\text { Parto natural OR educação continuada em enfermagem; enfermagem obstétrica } \\
\text { OR parto natural; enfermagem obstétrica AND educação continuada em } \\
\text { enfermagem; educação continuada em enfermagem OR parto natural. }\end{array}$ \\
\hline SCIELO & $\begin{array}{l}\text { Enfermagem obstétrica OR parto natural; enfermagem obstétrica OR educação } \\
\text { continuada em enfermagem; Enfermagem obstétrica } A N D \text { parto natural. }\end{array}$ \\
\hline BVS & $\begin{array}{l}\text { Enfermagem obstétrica AND parto natural; educação continuada em } \\
\text { enfermagem AND enfermagem obstétrica; educação continuada em } \\
\text { enfermagem AND parto natural. }\end{array}$ \\
\hline
\end{tabular}

Fonte: Garcia SA, et al., 2021.

\section{Análise dos dados}

A partir da coleta de dados, foram elencados 123 artigos, posteriormente submetidos à primeira etapa de análise, mediante a aplicabilidade dos critérios de inclusão. Os critérios foram: artigos de pesquisa publicados nos últimos cinco anos, artigos originais gratuitos e em periódicos disponíveis nas bases de dados selecionadas, nos idiomas de português, inglês e espanhol.

Ao final da primeira etapa de avaliação dos artigos, obteve-se a amostra de 76 estudos. Submetidos a uma nova análise que foi a leitura completa dos artigos para identificar aqueles que respondiam à pergunta problema dessa pesquisa, logrou-se de 23 artigos incluídos. O fluxograma especificando as etapas de pesquisa está exibido a seguir na Figura 1. 
Figura 1 - Fluxograma coleta e análise dos dados, 2021.
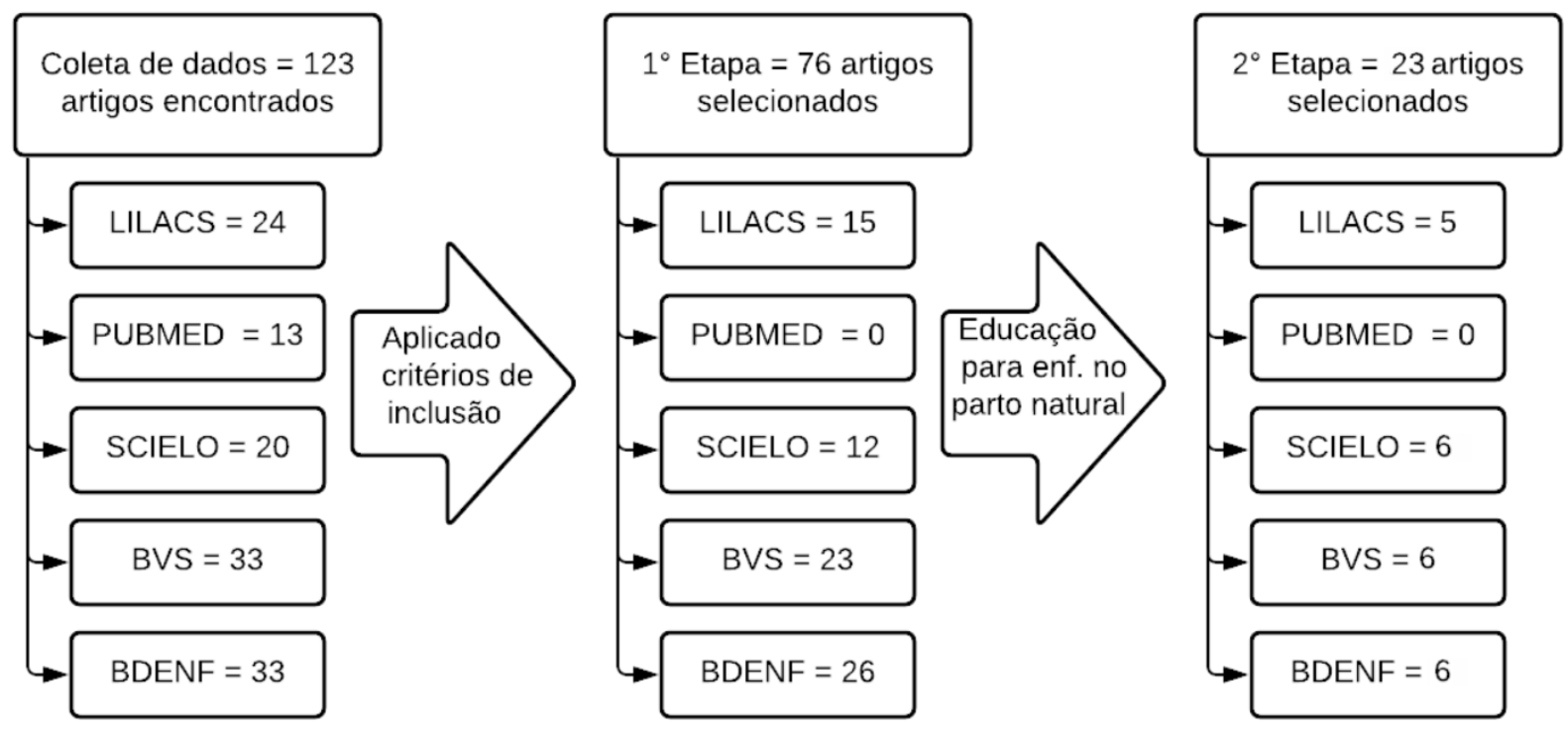

Fonte: Garcia SA, et al., 2021.

\section{RESULTADOS}

Elaborou-se, para facilitar a avaliação e a análise dos dados, um instrumento que pudesse fornecer informações detalhadas dos estudos (Quadro 1). Extraíram-se variáveis de identificação tais como: autores do estudo, título, objetivos, e resultado encontrado. Pretendeu-se com o instrumento, além de formar um banco de dados, mapear pontos pertinentes, integrar dados e caracterizar a amostra revisada.

Os resultados começam com a exposição do perfil dos artigos utilizados nesta etapa (Quadro 2). Evidenciaram-se duas categorias a partir dos resultados dos estudos selecionados: Parto natural e a assistência de enfermagem e defasagem na assistência: falta de educação continuada. 
Quadro 2 - Características dos estudos selecionados, 2021.

\begin{tabular}{|c|c|c|c|c|}
\hline № & Autor, Ano & Título & Periódico & Abordagem \\
\hline 1 & $\begin{array}{l}\text { Bomfim ANA, et } \\
\text { al., } 2021\end{array}$ & $\begin{array}{l}\text { Percepções de mulheres sobre a assistência de enfermagem durante o parto } \\
\text { normal. }\end{array}$ & $\begin{array}{l}\text { Revista Baiana de } \\
\text { Enfermagem }\end{array}$ & Qualitativa \\
\hline 2 & $\begin{array}{l}\text { Silva GF, et al., } \\
2021\end{array}$ & $\begin{array}{l}\text { Registros reflexivos na facilitação de processos de Educação Permanente } \\
\text { em Saúde. }\end{array}$ & Revista da SPAGESP & Qualitativa \\
\hline 3 & $\begin{array}{l}\text { Gama SGN, et al., } \\
2021\end{array}$ & $\begin{array}{l}\text { Atenção ao parto por enfermeira obstétrica em maternidades vinculadas à } \\
\text { Rede Cegonha, Brasil - } 2017 \text {. }\end{array}$ & $\begin{array}{l}\text { Revista Ciência \& Saúde } \\
\text { Coletiva }\end{array}$ & Qualitativa \\
\hline 4 & $\begin{array}{l}\text { Silva TM, et al., } \\
2020\end{array}$ & $\begin{array}{l}\text { Violência obstétrica: a abordagem da temática na formação de enfermeiros } \\
\text { obstétricos. }\end{array}$ & $\begin{array}{l}\text { Revista Acta Paul } \\
\text { Enfermagem }\end{array}$ & Descritiva / Qualitativa \\
\hline 5 & $\begin{array}{l}\text { Sanfelice CFO, et } \\
\text { al., } 2020\end{array}$ & $\begin{array}{l}\text { Curso de aprimoramento para enfermeiras obstétricas do Projeto ApiceOn: } \\
\text { relato de experiência. }\end{array}$ & Escola Anna Nery & Descritivo \\
\hline 6 & $\begin{array}{l}\text { Lira IMS, et al., } \\
2020\end{array}$ & $\begin{array}{l}\text { Intervenção educacional para melhoria na assistência ao trabalho de parto } \\
\text { normal. }\end{array}$ & Revista Enfermería Global & Experimental \\
\hline 7 & $\begin{array}{l}\text { Silva GF, et al., } \\
2020\end{array}$ & $\begin{array}{l}\text { Possibilidades para a mudança no modelo obstétrico hegemônico pelas } \\
\text { enfermeiras obstétricas }\end{array}$ & Revista Enfermagem UERJ & Qualitativa \\
\hline 8 & $\begin{array}{l}\text { Giacomini SM e } \\
\text { Hirsch ON, } 2020\end{array}$ & Parto "natural" e/ou "humanizado"? Uma reflexão a partir da classe. & Revista Estudos Feministas & Qualitativa \\
\hline 9 & $\begin{array}{l}\text { Vidal AT, et al., } \\
2020\end{array}$ & $\begin{array}{l}\text { Barreiras à implementação de recomendações ao parto normal no Brasil: a } \\
\text { perspectiva das mulheres. }\end{array}$ & $\begin{array}{l}\text { Revista Panamericana Salud } \\
\text { Publica }\end{array}$ & Qualitativa \\
\hline 10 & $\begin{array}{l}\text { Fontana RT, et al., } \\
2020\end{array}$ & $\begin{array}{l}\text { As metodologias usadas no ensino de enfermagem: com a palavra, os } \\
\text { estudantes. }\end{array}$ & Educação em Revista & Descritiva \\
\hline 11 & $\begin{array}{l}\text { Silva CM, et al., } \\
2020\end{array}$ & Interação social de puérperas com procedimentos invasivos no parto. & Revista Enfermagem UERJ & Qualitativa \\
\hline 12 & $\begin{array}{l}\text { Lima MM, et al., } \\
2020\end{array}$ & Enfermeiras obstétricas no processo de parturição: percepção das mulheres. & Revista de Enfermagem UERJ & Qualitativa \\
\hline
\end{tabular}




\begin{tabular}{|c|c|c|c|c|}
\hline № & Autor, Ano & Título & Periódico & Abordagem \\
\hline 13 & $\begin{array}{l}\text { Pereira LR, et al., } \\
2020\end{array}$ & Parto normal e Intervenções ocorridas em uma maternidade pública. & $\begin{array}{l}\text { Revista Baiana de } \\
\text { Enfermagem }\end{array}$ & Qualitativa \\
\hline 14 & $\begin{array}{l}\text { Vilela AT, et al., } \\
2019\end{array}$ & Percepção dos enfermeiros obstetras diante do parto humanizado & $\begin{array}{l}\text { Revista de Enfermagem UFPE } \\
\text { Online }\end{array}$ & Descritiva / Qualitativa \\
\hline 15 & $\begin{array}{l}\text { Camacho NPR, et } \\
\text { al., } 2019\end{array}$ & $\begin{array}{l}\text { Conhecimento e aplicabilidade dos métodos não farmacológicos utilizados } \\
\text { pelos enfermeiros obstetras para alívio da dor no trabalho de parto. }\end{array}$ & Revista Nursing & Qualitativa \\
\hline 16 & $\begin{array}{l}\text { Silva TPR, et al., } \\
2019\end{array}$ & $\begin{array}{l}\text { Enfermagem obstétrica nas boas práticas da assistência ao parto e } \\
\text { nascimento }\end{array}$ & $\begin{array}{l}\text { Revista Brasileira de } \\
\text { Enfermagem }\end{array}$ & Transversal \\
\hline 17 & $\begin{array}{l}\text { Carlos GAV, et al., } \\
2019\end{array}$ & $\begin{array}{l}\text { Perfil dos participantes de um curso de aprimoramento em enfermagem } \\
\text { obstétrica }\end{array}$ & $\begin{array}{l}\text { Revista Mineira de } \\
\text { Enfermagem }\end{array}$ & Transversal \\
\hline 18 & $\begin{array}{l}\text { Alves TTM, et al., } \\
2018\end{array}$ & Atuação de a enfermeira obstetra no desenrolar do trabalho de parto e parto & $\begin{array}{l}\text { Revista de Enfermagem e } \\
\text { Atenção à Saúde }\end{array}$ & Descritiva / Qualitativa \\
\hline 19 & $\begin{array}{l}\text { Sá ACMGN, et al., } \\
2018\end{array}$ & $\begin{array}{l}\text { Contribuições da educação permanente para qualificação da assistência de } \\
\text { enfermagem em um hospital público. }\end{array}$ & $\begin{array}{l}\text { Revista Brasileira de Ciências } \\
\text { da Saúde }\end{array}$ & $\begin{array}{l}\text { Qualitativa / } \\
\text { Quantitativa }\end{array}$ \\
\hline 20 & Lima F, et al., 2018 & $\begin{array}{l}\text { Educação permanente em saúde como fortalecimento da enfermagem } \\
\text { obstétrica. }\end{array}$ & $\begin{array}{l}\text { Revista de Enfermagem UFPE } \\
\text { Online }\end{array}$ & Descritiva / Qualitativa \\
\hline 21 & $\begin{array}{l}\text { Andrade LO, et al., } \\
2017\end{array}$ & Prática dos profissionais de enfermagem diante do parto humanizado. & $\begin{array}{l}\text { Revista de Enfermagem UFPE } \\
\text { Online }\end{array}$ & Descritiva / Qualitativa \\
\hline 22 & $\begin{array}{l}\text { Quental LLC, et } \\
\text { al., } 2017\end{array}$ & Práticas educativas com gestantes na Atenção Primária à Saúde. & $\begin{array}{l}\text { Revista de Enfermagem UFPE } \\
\text { Online }\end{array}$ & Revisão Integrativa \\
\hline 23 & $\begin{array}{l}\text { Freire HSS, et al., } \\
2017\end{array}$ & $\begin{array}{l}\text { Parto normal assistido por enfermeira: experiência e satisfação de } \\
\text { puérperas. }\end{array}$ & $\begin{array}{l}\text { Revista de Enfermagem UFPE } \\
\text { Online }\end{array}$ & Descritiva / Qualitativa \\
\hline
\end{tabular}

Fonte: Garcia SA, et al., 2021. 


\section{DISCUSSÃO}

O trabalho de parto acontecia naturalmente respeitando todos os aspectos fisiológicos do corpo da mulher, com o avanço da sociedade e da medicina houve a institucionalização do parto, passando o mesmo a ser visto como um evento patológico, onde não havia respeito à autonomia da mulher, levando-a a ser submetida a intervenções desnecessárias e procedimentos tidos como dolorosos (GAMA SGN, et al., 2021; SILVA CM, et al., 2020).

Trabalho de parto e nascimento são experiências marcantes na vida de uma mulher que podem vir acompanhadas dos mais diversos sentimentos, por serem momentos imprevisíveis e desconhecidos, surgem em um misto de expectativas, preocupações, ansiedades, medos e angústias. Por isso, os profissionais envolvidos, além de oferecer uma atenção cercada de conhecimentos técnico-científicos, devem buscar entender as percepções e individualidades, oferecendo uma assistência humanizada que permite a participação da mulher no processo de parto e nascimento para que ocorra da forma mais natural possível (FREIRE HSS, et al., 2017).

A assistência do profissional de Enfermagem Obstétrica é essencial para a realização de um parto humanizado e assistencial, pois, além dos conhecimentos científicos, necessita reconhecer a mulher como um ser singular, criando vínculos de confiança, apoio e transmitindo tranquilidade, deixando a mulher atuar, durante o parto, como protagonista. É de extrema importância que os enfermeiros obstetras sejam capacitados e dispostos a prestar tais cuidados e terem iniciativas que respeitem a fisiologia do parto e a autonomia da mulher (VILELA AT, et al., 2019).

Atualmente, observa-se a busca pela reversão desse processo de institucionalização do parto, com o ideal de torná-lo o mais humanizado possível, priorizando assistência a todos os aspectos que envolvem a mulher: espirituais, psicológicos, sociais e biológicos (ALVES TTM, et al., 2018). A essência da humanização do parto natural apresenta como práticas importantes: exercícios com bola suíça, cavalinho, massagem lombossacral, banho de imersão e aquecido, realização de monitorização fetal, orientação e apoio emocional, e pôr fim a presença de acompanhante (CAMACHO ENPR, et al., 2019).

Observou-se no estudo realizado em uma maternidade no Estado do Ceará que as práticas de humanização permitem que o parto seja realizado da forma mais natural possível, dando autonomia a mulher, assim também obtendo resultados perinatais, com bebês em boa saúde e mães satisfeitas. Os profissionais devem ter cuidado para transmitir segurança e confiança as parturientes, devido a carência de informações e conhecimento (FREIRE HSS, et al., 2017).

Muitas mulheres sentem do parto normal devido ao grande número de intervenções, muitas vezes desnecessárias, que cerca a assistência obstétrica: enema, jejum prolongado, acesso venoso, medicamentos indutores do trabalho de parto, manobra de kristeller, dentre outras. Dessa forma, acarretam em partos difíceis, traumáticos com sequelas comportamentais e emocionais que geram inseguranças e questionamentos na capacidade das mulheres parirem, contribuindo e influenciados no aumento do índice de cesariana nos últimos anos no Brasil (BOMFIM ANA, et al., 2021).

O aumento da enfermagem obstétrica faz com que as taxas de intervenções sejam reduzidas, e assim possibilitando um maior índice de partos vaginais, através de práticas úteis e reduzindo também práticas que se faz prejudiciais a binômio mãe e filho. Faz com que o ato de parir seja mais natural possível, trazendo à tona a fisiologia e deixando-a agir. A enfermagem deve favorecer e incentivar a atuação e protagonismo da mulher no seu processo de parturição (SILVA TPR, et al., 2019).

Contudo, o Ministério da Saúde tem desenvolvido ações para incentivar a formação e aprimoramento da enfermagem, para que haja a atuação efetiva e ativa na mudança do modelo obstétrico atual, por meio de programas de residência e cursos de especialização em enfermagem obstétrica (SILVA GF, et al., 2020; CARLOS GAV, et al., 2019).

Educação continuada são práticas que envolve conjunto de ações que associam experiência e aprendizagem com o objetivo de desenvolver a educação como um processo facilitador para o alcance da 
saúde. Portanto, os profissionais de enfermagem devem possuir uma relação pautada no diálogo e na valorização da individualidade, tornando, dessa forma, necessário o conhecimento das técnicas educativas, por parte destes profissionais para reconstrução da prática e saber. Exercendo seus conhecimentos em diferentes contextos, sendo um deles a educativa, entendida como principal estratégia para promoção da saúde (QUENTAL LLC, et al., 2017).

Compreendendo que é uma aprendizagem importante e constante para equipe de enfermagem visto que, o conhecimento fornece uma assistência de qualidade e pautada na humanização. Além, de oferecer uma abordagem mais ampla promovendo uma maior conexão através da forma teórica e prática. Diante disso a educação continuada é de extrema importância visto que são práticas utilizadas com objetivo de desenvolvimento pessoa e profissional, buscando aprimorar seus conhecimentos. (LIMA F, et al., 2018).

A enfermagem obstétrica também tem um importante papel como educador da parturiente, de sua família e acompanhante, orientando e informando os acerca do trabalho de parto, dando-Ihes apoio tanto físico como emocional. Para que um profissional seja considerado como qualificado deve-se ter recebido formação e treinamento, devido a exigência de competências sobre as diferentes fases do trabalho de parto, além de ter um bom manejo com as práticas necessárias ao parto normal (LIRA IMS, et al., 2020).

Desse modo, a educação continuada tem a finalidade de desenvolver profissionais capacitados a envidarse na promoção integral a saúde, mediante as ações educativas a serem desenvolvidas com as parturientes, viabilizando a troca de saberes e promoção da saúde, tendo como finalidade atuação mais efetiva e eficaz contribuindo para o fortalecimento do profissional da enfermagem nessa pratica (SILVA TM, et al., 2020).

Diante disso, a educação continuada visa profissionais preparados e comprometidos de forma pessoal e profissional, que recebam a mulher com respeito, ética e dignidade, incentivando a exercer a sua liberdade no resgate de seu papel ativo no processo parturição, a além repudiar qualquer tipo de discriminação e violência que possa comprometer os seus direitos de cidadania (ANDRADE LO, et al., 2017).

A educação em saúde é compreendida como uma inspiração para mudanças positivas de trabalho em equipe, com o objetivo de implementar práticas embasada em evidências científicas estabelecendo metas, objetivos e desafios para a equipe ajuda humanizar o atendimento. A enfermagem obstétrica é uma categoria em asserção, que vem ocupando cada vez mais espaço no cenário do trabalho de parto natural (SANFELICE CFO, et al., 2020).

\section{Parto natural e a assistência de enfermagem}

O parto natural é aquele que ocorre livre de intervenções e em conjunto com a humanização da assistência ao parto, que prioriza a autonomia da mulher, respeitando sua fisiologia corporal, tempo de trabalho de parto e todo seu processo, proporcionando apoio psicológico, emocional, físico e orientando a parturiente (GIACOMINI SM e HIRSCH ON, 2020; VIDAL AT, et al., 2020).

O parto é um processo natural e fisiológico, que cada mulher sente de uma maneira diferente, visto que, é um misto de emoções e grandes mudanças, o profissional de enfermagem deve estar capacitado acolher essa mulher fisicamente e emocionalmente, prestando uma assistência de qualidade e humanizada, adaptando a individualidade dessa mulher, na qual exista uma relação horizontalizada, onde são compartilhadas decisões e informações (ANDRADE LO, et al., 2017).

O uso da assistência humanizada pela equipe de enfermagem reduz à utilização de intervenções desnecessárias que trazem malefícios as parturientes (PEREIRA LR, et al., 2019). A assistência da enfermagem obstétrica é crucial, visto que, contribui para melhoria da qualidade do trabalho de parto, por meio da inclusão da mulher na tomada de decisão. A essência da enfermagem se sobressai a uso de procedimentos técnicos (BOMFIM ANA, et al., 2021).

\section{Defasagem na assistência: falta de educação continuada}

A enfermagem tem sua assistência baseada em conhecimentos técnicos científicos, que constantemente são atualizados, fazendo-se necessários profissionais críticos e reflexivos que buscam o aprimoramento de 
suas habilidades e discernimento periodicamente (FONTANA RT, et al., 2020). É necessário conciliar teoria e prática desde a graduação, formação e posteriormente por meio de programas de educação continuada, é obrigação de o enfermeiro manter-se atualizado (SILVA GM e GUANAES-LORENZI C, 2021).

A conscientização da enfermagem sobre o trabalho de parto natural, ofertando assistência integral, empática e com boa comunicação é perfeitamente possível a partir da implementação de um sistema assistencial que proporciona experiência positiva sobre o parto (LIMA MM, et al., 2020). Uma visão pautada no entendimento e satisfação mediante a qualidade propiciada pela educação continuada instituída pelo Ministério da Saúde que estimula a atualização constante do conhecimento (SÁ ACMGN, et al., 2018).

A Educação continuada busca aprimorar conhecimento por meio de desenvolvimento profissional contínuo, se aperfeiçoando na sua profissão agregando suas habilidades, dispondo de uma visão concreta do meio de atuação que está inserido. Essa prática permite que a equipe de enfermagem continue em constante processo educativo no qual reflete na qualidade do auxílio proporcionando boas práticas (LIRA IMS, et al., 2020).

\section{CONSIDERAÇÕES FINAIS}

A realização deste estudo possibilitou descrever a importância da educação continuada em enfermagem, conceituar e entender as particularidades do parto natural. Diante do estudo constatou-se que são necessários projetos que incentivem e facilitem o acesso à educação continuada no tocante da enfermagem visto que é o principal meio de transformação da atuação da enfermagem no ambiente obstétrico, pois, profissionais de enfermagem qualificados melhoram a qualidade de sua assistência prestada, desenvolvem habilidades de comunicação mais eficiente, tem sua prática baseada em conhecimento científico e o resultado deste conjunto de ações é satisfação da parturiente na experiência do parto, além de contribuir para o crescimento da categoria na prática obstétrica.

\section{REFERÊNCIAS}

1. ALVES TTM, et al. Atuação da enfermeira obstetra no desenrolar do trabalho de parto e parto. Rev. Enferm. Atenção Saúde, 2018; 7(1): 41-50.

2. ANDRADE LO, et al. Práticas dos profissionais de enfermagem diante do parto humanizado. Rev. Enferm. UFPE online, 2017; 11(supl.6): 2576-2585.

3. ARAÚJO BRO, et al. Entre ritos e contextos: Decisões e significados atribuídos ao parto natural humanizado.Cult. Cuid, 2019; 23(54): 206-216.

4. BOMFIM ANA, et al. Percepções de mulheres sobre a assistência de enfermagem durante o parto normal. Rev. Baiana Enferm. 2021; 35: e39087.

5. CAMACHO ENPR, et al. Conhecimento e aplicabilidade dos métodos não farmacológicos utilizados pelos enfermeiros obstetras para alívio da dor no trabalho de parto. Nursing, 2019; 22(257): 3193-3198.

6. CARLOS GAV, et al. Perfil dos participantes de um curso de aprimoramento em enfermagem obstétrica. REME Rev. Min. Enferm. 2019; 23: e1153.

7. FONTANA RT, et al. As metodologias usadas no ensino de enfermagem: com a palavra, os estudantes. Educ. Rev. 2020; 36: e220371.

8. FREIRE HSS, et al. Parto normal assistido por enfermeira: experiência e satisfação de puérperas. Rev. Enferm. UFPE online, 2017; 11(6): 2357-2367.

9. FREITAS PF, FERNANDES TMB. Associação entre fatores institucionais, perfil assistencial ao parto e taxas de cesárea em Santa Catarina. Rev. Bras. Epidemiol. 2016; 19 (3): 525-538.

10. GAMA SGN, et al. Atenção ao parto por enfermeira obstétrica em maternidades vinculadas à Rede Cegonha, Brasil - 2017. Ciênc. Saúde Coletiva, 2021; 26(3): 919-929.

11. GIACOMINI SM, HIRSCH ON. Parto natural e/ou humanizado? Uma reflexão a partir da classe. Rev.Estud. Fem, 2020; 28 (1): e57704.

12. LIMA $F$, et al. Educação permanente em saúde como fortalecimento da enfermagem obstétrica. Rev. Enferm. UFPE online, 2018; 12(2): 391-397.

13. LIMA MM, et al. Enfermeirasobstétricas no processo de parturição: percepção das mulheres. Rev. Enferm. UERJ, 2020; 28: e45901.

14. LIRA IMS, et al. Intervenção educacional para melhoria na assistência ao trabalho de parto normal. Enferm. Glob. 2020; 19(58): 226-256. 
15. OLIVEIRA PS, et al. Boas práticas no processo de parto: concepções de enfermeiras obstétricas. Rev. Bras. Enfermagem, 2019; 72(2): 455-462.

16. PEREIRA LR, et al. Parto normal e intervenções ocorridas em uma maternidade pública. Rev. Baiana Enferm. 2019; 33: e32631.

17. POSSATI AB, et al. Humanização do parto: significados e percepções de enfermeiras. Esc. Anna Nery, 2017; 21(4): e20160366.

18. QUENTAL LLC, et al. Práticas educativas com gestantes na Atenção Primária à Saúde. Rev. Enferm. UFPE online, 2017; 11 (supl.12): 5370-5381.

19. SÁ ACMGN, et al. Contribuições da educação permanente para qualificação da assistência de enfermagem em um hospital público. Rev. Bras. Ciênc. Saúde, 2018; 22(1): 87-94.

20. SANFELICE CFO, et al. Curso de aprimoramento para enfermeiras obstétricas do Projeto Apice On: relato de experiência. Esc. Anna Nery Rev. Enferm. 2020; 24(2): e20190212.

21. SILVA CM, et al. Interação social de puérperas com procedimentos invasivos no parto. Rev. Enferm. UERJ, 2020; 28: e52496.

22. SILVA CPG, et al. Da educação em serviço à educação continuada em um hospital federal. Esc. Anna Nery, 2020; 24(4): e20190380.

23. SILVA EA, et al. Conhecimento de puérperas sobre boas práticas em centro de parto Rev. Enferm. UFPE online, 2021; 15(1): 1-14.

24. SILVA GF, et al. Possibilidades para a mudança do modelo obstétrico hegemônico pelas enfermeiras obstétricas. Rev. Enferm. UERJ, 2020; 28: e49421.

25. SILVA GM, GUANAES-LORENZI C. Registros reflexivos na facilitação de processos de Educação Permanente em Saúde. Rev. SPAGESP, 2021; 22(1): 6-21.

26. SILVA TM, et al. Violência obstétrica: a abordagem da temática na formação de enfermeiros obstétricos. Acta Paul. Enferm. 2020; 33: eape20190146.

27. SILVA TPR, et al. Enfermagem obstétrica nas boas práticas da assistência ao parto e nascimento. Rev. Bras. Enferm. 2019; 72(Suppl3): 235-242.

28. SOUZA MT, et al. Revisão integrativa: o que é e como fazer. Einstein, 2010; 8(1): 102-106.

29. VIDAL AT, et al. Barreiras à implementação de recomendações ao parto normal no Brasil: a perspectiva das mulheres. Rev. Panam. Salud. Publica, 2020;44:e164.

30. VILELA AT, et al. Percepção dos enfermeiros obstetras diante do parto humanizado. Rev. Enferm. UFPE online, 2019; 13: 1-6. 